\title{
PSEUDO-RIEMANNIAN GEOMETRY CALIBRATES OPTIMAL TRANSPORTATION
}

\author{
Young-Heon Kim, Robert J. McCann and Micah Warren
}

\begin{abstract}
Given a transportation cost $c: M \times \bar{M} \rightarrow \mathbf{R}$, optimal maps minimize the total cost of moving masses from $M$ to $\bar{M}$. We find, explicitly, a pseudo-metric and a calibration form on $M \times \bar{M}$ such that the graph of an optimal map is a calibrated maximal submanifold, and hence has zero mean curvature. We define the mass of spacelike currents in spaces with indefinite metrics.
\end{abstract}

\section{Introduction}

The aim of this article is to adapt the notion of calibration (see [5] [11]) to a pseudo-Riemannian framework constructed to describe and explore the geometry of optimal transportation from a new perspective.

Given a smooth function $c: M \times \bar{M} \rightarrow \mathbf{R}$ (called the transportation cost), and probability densities $\rho$ and $\bar{\rho}$ on two manifolds $M$ and $\bar{M}$ (possibly with boundary), a natural variational problem is to find an optimal map $F: M \rightarrow \bar{M}$ that minimizes the total cost

$$
\int_{M} c(x, F(x)) \rho(x) d x
$$

under the constraint that for every measurable function $f$ on $\bar{M}$,

$$
\int_{M} f(F(x)) \rho(x) d x=\int_{\bar{M}} f(\bar{x}) \bar{\rho}(\bar{x}) d \bar{x} .
$$

The last condition will be denoted by $F_{\#} \rho=\bar{\rho}$, in which case we say $F$ pushes $\rho$ forward to $\bar{\rho}$. This variational problem, called optimal transportation, dates back to Monge in 18th century and is currently undergoing a rapid and broad development, especially in relation to geometry; see Villani's recent book and the references it contains [13].

Received by the editors April 8. 2010.

R.J.M.'s research was supported in part by Natural Sciences and Engineering Research Council of Canada Grant 217006-08 and United States National Science Foundation Grant DMS-0354729. Y-H.K. is supported partly by NSF Grant DMS-0635607 (through the membership at Institute for Advanced Study) and NSERC discovery grant 371642-09. M.W. is supported in part by NSF Grant DMS-0901644. Any opinions, findings and conclusions or recommendations expressed in this material are those of authors and do not reflect the views of either Natural Sciences and Engineering Research Council of Canada or the US National Science Foundation. 
We introduce a metric on $M \times \bar{M}$ via the following symmetric bilinear form on $T M \times T \bar{M}$, defined on a coordinate frame:

$$
h_{c}^{\rho, \bar{\rho}}=\left(\frac{\rho(x) \bar{\rho}(\bar{x}))}{|\operatorname{det}(D \bar{D} c(x, \bar{x}))|}\right)^{\frac{1}{n}}\left(\begin{array}{cc}
0 & -D \bar{D} c \\
-(D \bar{D} c)^{T} & 0
\end{array}\right)
$$

and calibration

$$
\Phi(x, \bar{x})=\frac{\rho(x) d x+\bar{\rho}(\bar{x}) d \bar{x}}{2} .
$$

Here $D \bar{D} c$ denotes second derivatives of the cost function in mixed directions. Our central result, Theorem 3.10 can be stated imprecisely as follows:

Theorem 1.1. Under reasonable conditions, the graph of the optimal map is calibrated by $\Phi$, and is thus a stable maximal surface with respect to the pseudo-metric $h_{c}^{\rho, \bar{\rho}}$ on $M \times \bar{M}$.

A new geometric aspect of optimal transportation was observed by the first two authors [7]. Namely, the transportation cost $c$ induces a certain pseudo-metric on $M \times \bar{M},(3.2)$, in such a way that positivity of its Riemannian curvature tensor on certain sections gives a necessary condition for the regularity of general optimal maps $F$. This result gives a geometric perspective on the fundamental regularity theory developed for optimal maps by Ma, Trudinger and Wang [9] [12] and Loeper [8]. Moreover, the graph of the optimal map $F$ is a Lagrangian submanifold with respect to the Kähler form of the pseudo-metric (see Section 3 or [7] for more details).

Mealy [11] developed the idea of calibration on pseudo-Riemannian manifolds, and introduced the special Lagrangian equations for signature $(n, n)$ metrics:

$$
\sum_{k=0}^{\lfloor(n-1) / 2\rfloor} \sigma_{2 k+1}\left(D^{2} \phi\right)=0 \quad \text { (here, } \sigma_{k} \text { denotes the } k \text {-symmetric function). }
$$

These describe functions whose gradient graphs $(x, \nabla \phi(x))$ are maximal surfaces in the "standard" pseudo-Euclidean space, $\left(\mathbb{R}^{n+n}, d x^{2}-d y^{2}\right)$, provided that

$$
\left|D^{2} \phi\right|<1
$$

which is equivalent to the graph being spacelike. After Hitchin [6, Prop. 3] connected Monge-Ampère equations and special Lagrangian submanifolds in a pseudoEuclidean space, the third author [15] explored these equations, which essentially describe Mealy's submanifolds under a coordinate change. In Hitchin's coordinate setting the gradient map of a convex function $\phi$ solving the standard Monge-Ampère equation

$$
\operatorname{det} D^{2} \phi=1,
$$

gives a calibrated submanifold in the product space $\mathbf{R}^{n} \times \mathbf{R}^{n}$ equipped with a pseudoEuclidean metric, $d x \cdot d y$. In fact, Hitchin's definition of special Lagrangian is that 
a linear combination of certain volume forms vanish along a Lagrangian submanifold. Our calibration (1.4) encodes precisely this definition for the volume forms $\rho d x$ and $\bar{\rho} d \bar{x}$.

In this paper, we provide a framework that combines and extends these results by introducing the pseudo-metric defined in (1.3), which is conformally equivalent to the pseudo-metric in [7] but depends on $\rho$ and $\bar{\rho}$ in addition to $c$. With respect to this metric, the graphs of optimal maps are calibrated, thus give maximal submanifolds (see Theorem 3.10 and Corollary 3.11). This demonstrates how the functional extremality of optimal maps for (1.1)-(1.2) characterizes in a natural way the geometric extremality of their graphs.

Mealy's calibrations compare admissible (spacelike, in our case of interest) smooth submanifolds to other admissible smooth submanifolds, but we would like a more general setting allowing for spacelike submanifolds which are merely rectifiable.

Solutions to the optimal transportation problem are not in general smooth. Allowing for nonsmooth solutions, we will use the language of currents, which requires that we verify that the submanifolds in question are rectifiable. This has been shown recently in [10], given assumption (A2), using the Lewy transformation, which returns us to a situation which locally looks like Mealy's, where the submanifolds in question are automatically Lipschitz graphs. The development of currents generalizes some results for the Euclidean cost function appearing in [1]. The behavior of solutions to the problem of optimal transportation for general measures on general metric spaces can be quite wild. It is unclear how much of this theory can be adapted to more general cases.

Our use of currents will require some apparently new definitions of mass for currents in a pseudo-Riemannian manifold, see definition 2.1. While the corresponding notions of mass from Euclidean geometric measure theory are expressed in terms of suprema, we will need to express mass in terms of infima, and will also need some sort of space orientation for these definitions to make sense. These definitions recover the volume of a smooth spacelike submanifold.

After laying down some pseudo-Riemannian geometric measure theory in section 2 , we will define calibrations on spaces with pseudo-metrics. In the final section we show how the optimal transportation problem fits into this setting.

The calibrated submanifolds with the metric we are considering are not in general graphs of the optimal maps, rather these are graphs of Lie solutions as developed by Delanoe [2], see section 4 . The moduli space of such calibrated submanifolds is discussed in [14] .

\section{Spacelike Currents in an Indefinite (pseudo) Metric}

We formulate some definitions which adapt the geometric measure theoretic notion of mass to oriented manifolds with indefinite metric. This notion will allow us to compare the mass of calibrated currents to homologous currents in Section 3. 
Let $\left(N^{n, m}, h, \tau\right)$ denote a smooth $(n+m)$-dimensional manifold, a metric with signature $(n, m)$ and a space orientation $n$-form $\tau$. An oriented $n$-plane $P$ is spacelike if $\left.h\right|_{P}>0$, and is $\tau$-oriented if $\tau(\xi)>0$ for any $n$-vector $\xi \in \Lambda^{n} T_{x} N$ representing $P$. Let $G_{+}\left(n, T_{x} N\right)$ denote the collection of $n$-vectors which are both spacelike and $\tau$-oriented. (To be clear, $G_{+}$is a cone, not a Grassmannian.) Note that the "unit sphere" of $n$-vectors in an indefinite metric will have distinct connected components, since each such plane comes with two orientations distinguished by the sign of the space orientation form $\tau$ (see Proposition 2.2). Here, as in many other of our arguments, it is crucial that $n$ is the maximum dimension of any spacelike plane. An element $\xi \in \Lambda^{n} T_{x} N$ is called simple if it is a single product of $n$ vectors, i.e. $\xi=v_{1} \wedge \ldots \wedge v_{n}$. For a simple $\xi \in G_{+}\left(n, T_{x} N\right)$ we will be interested in the $h$-norm of $\xi$, which is the $n$-dimensional $h$-volume defined by

$$
\|\xi\|_{h}:=\sqrt{\operatorname{det}\left(h\left(v_{i}, v_{j}\right)\right)_{1 \leq i, j \leq n}}
$$

In the following, a current shall mean a de Rham $n$-current with compact support on $N$, that is, an element of the dual space $\left(\Omega^{n} N\right)^{*}$ of the space $\Omega^{n} N$ of smooth $n$-forms on $N$. Recall that a compact oriented $n$-submanifold with locally finite Hausdorff measure defines a linear functional on the space of $n$-forms by integration, thus a "reasonable" nonsmooth submanifold is also a current, in a similar way. The support of current $T$ is given by the following: Let $U$ be the largest open set having the property that $T(\varphi)=0$ whenever $\varphi$ is compactly supported inside $U$. The support of $T, \operatorname{spt}(T)$, is the complement of $U$.

Definition 2.1 (Mass of a current; comass of a form). Define the set of simple $\tau$-oriented space-like unit $n$-planes by

$$
\mathcal{P}_{x}=\left\{\xi \in \Lambda^{n} T_{x} N\left|\xi=v_{1} \wedge \ldots \wedge v_{n}, \tau(\xi)>0, h\right|_{\operatorname{span}\left(v_{1}, \ldots, v_{n}\right)}>0,\|\xi\|_{h}=1\right\} .
$$

Define the oriented comass of an $n$-form $\psi_{x}$ at a point via

$$
\left\|\psi_{x}\right\|_{h}^{*}=\inf _{\xi \in \mathcal{P}_{x}} \psi_{x}(\xi)
$$

On any set $U \subset N$, define the oriented comass of a $n$-form $\psi$ on $U$ as

$$
\|\psi\|_{(U, h)}^{*}=\inf _{x \in U}\left\|\psi_{x}\right\|_{h}^{*} .
$$

Now define the oriented mass of a current $T \in\left(\Omega^{n} U\right)^{*}$ by

$$
\|T\|_{(U, h)}=\inf _{\|\psi\|_{(U, h)}^{*} \geq 1} T(\psi) .
$$

Some remarks about the definitions: First, since the space $\mathcal{P}_{x}$ is noncompact, we observe that the values of all of the infima in Definition 2.1 may be $-\infty$. Time-like or negatively oriented planes are given infinite weight, thus any current with enough time-like or negatively oriented planes is given a mass of $-\infty$. Fortunately, this rules out certain pathologies that occur for pseudo-metrics (see examples in [15, section $4]$ ), and recovers expected values of mass for smooth space-like sets. 
For Riemannian (thus positive definite) metrics, a calibration is a closed $p$-form $\Psi$ such that for all $p$-vectors $\xi, \Psi_{x}(\xi) \leq\|\xi\|$ (see [5]). A calibrated current $S$ is one for which $S(\Psi)=\|S\|$. It follows from Stokes' theorem that smooth calibrated manifolds are minimal. In a pseudometric, a calibration needs to give the opposite inequality. To be precise, we define:

Definition 2.2 (Calibration for indefinite metric). A calibration on $\left(N^{n, m}, h, \tau\right)$ is an $n$-form $\Psi$ such that

$$
d \Psi=0
$$

and

$$
\Psi_{x}(\xi) \geq\|\xi\|_{h}
$$

at each point $x$, for each space-like tangent plane $\xi$. Notice that this latter condition is equivalent to $\|\Psi\|_{h}^{*} \geq 1$.

Proposition 2.1. Let $S$ be a smooth, oriented, compact n-dimensional space-like submanifold of $N$. Let $\operatorname{vol}_{h}(S)$ denote the volume of $S$ with respect to the pseudometric $h$. Then, $\operatorname{vol}_{h}(S)=\|S\|_{(N, h)}$.

Proof. At each $x$, for a given spacelike simple $n$-vector $\xi \in \Lambda^{n} T_{x} N$, the form given by the $h$-inner product with $\xi$ has comass 1 (See [11, pg 797]). This form depends smoothly on the tangent space to $S$. (The corresponding simple $n$-vector field is obtained by choosing a smooth frame field and taking the $n$-product of the basis vectors.) Thus we can find a form $\psi \in \Omega^{n} S$ such that for each $x \in S, \psi_{x}(\varsigma) \geq\|\varsigma\|_{h}$ for all $\varsigma \in \mathcal{P}_{x}$ with equality when and only when $\varsigma$ represents the oriented tangent plane to $S$. Extend this smoothly to a form $\psi_{0}$ which has positive comass on a neighborhood $V_{0}$ of $S$. Dividing by the pointwise comass at each point where the comass is positive, we have that $\left\|\psi_{0}\right\|_{\left(V_{0}, h\right)}^{*}=1$. Next, take a cover of $N$ with open sets $V_{i}$, so that $V_{i} \cap S=\emptyset$, for $i \geq 1$, and so that on each $V_{i}$, there is a $\psi_{i}$ with $\left\|\psi_{i}\right\|_{\left(V_{i}, h\right)}^{*} \geq 1$. Such $V_{i}$ are easily locally available. Noting that the comass is superadditive, we may sum over a partition of unity and get a form, which we call $\psi$, with $\|\psi\|_{(N, h)}^{*} \geq 1$. Also, for any $\varphi$ with $\|\varphi\|_{(N, h)}^{*} \geq 1, S(\varphi) \geq S(\psi)$, by the choice of $\psi$. It follows that

$$
\inf _{\|\varphi\|_{(N, h)}^{*} \geq 1} S(\varphi)=S(\psi)=\int_{S} \psi=\int_{S} d \operatorname{vol}_{h}(S)
$$

This completes the proof.

From the above proposition, it is legitimate to use the notion of mass of currents to compare volumes of smooth space-like submanifolds.

We conclude this section with a remark:

Proposition 2.2. Let $h$ be an indefinite metric on $\mathbb{R}^{n+m}$ of the form $d x^{2}-d y^{2}$. The "unit sphere" $\|\xi\|_{h}=1$ of space-like $n$-planes is disconnected. 
Proof. Let $\xi$ be an $n$-plane given by $v_{1} \wedge \cdots \wedge v_{n}$. The projection $\pi$ of the independent set $\left\{v_{i}\right\}$ onto $\mathbb{R}^{n}$ gives an independent set, otherwise the plane would contain time-like or null vectors. Define $\tau(P)=\operatorname{det}\left(\pi\left(v_{1} \wedge \cdots \wedge v_{n}\right)\right)$. It is clear that the alternating form $\tau$ will be either positive or negative on any space-like plane.

\section{Application to Optimal Transportation}

Let $M, \bar{M}$ be oriented $n$-dimensional manifolds and let $\rho, \bar{\rho}$ be smooth volume forms on $M, \bar{M}$, respectively, with $\int_{M} \rho=\int_{\bar{M}} \bar{\rho}=1$. In oriented local coordinates $\left(x^{1}, \cdots, x^{n}, \bar{x}^{1}, \cdots \bar{x}^{n}\right)$ of $(x, \bar{x}) \in M \times \bar{M}$, we have the expression $\rho=\rho(x) d x, \bar{\rho}=$ $\bar{\rho}(\bar{x}) d \bar{x}, \rho(x), \bar{\rho}(\bar{x})>0$, where $d x=d x^{1} \wedge \cdots \wedge d x^{n}, d \bar{x}=d \bar{x}^{1} \wedge \cdots \wedge d \bar{x}^{n}$. Define an $n$-form $\Phi$ on $M \times \bar{M}$ in local coordinates (see upcoming subsection 3.1) by

$$
\Phi(x, \bar{x})=\frac{\rho(x) d x+\bar{\rho}(\bar{x}) d \bar{x}}{2} .
$$

Notice that we could write this in the invariant form

$$
\Phi=\frac{\pi^{*} \rho+\bar{\pi}^{*} \bar{\rho}^{*}}{2}
$$

where $\pi^{*}, \bar{\pi}^{*}$ are pullbacks of the projection mapping to $M$ and $\bar{M}$ respectively. In this section, we show the compactly supported portions of the graph of an optimal map are calibrated by the form $\Phi$, hence maximal with respect to the pseudo-metric $h^{\rho, \bar{\rho}}$ in metric.

Let $c: M \times \bar{M} \rightarrow \mathbf{R}$ be a positive, continuous, superdifferentiable cost function which is smooth on a set $N=(M \times \bar{M})$ - $\mathfrak{C}$, where $\mathfrak{C}$ is a closed measure zero set which we will call the "cut locus". (The reason for the terminology "cut-locus" is clear if the cost function is the distance squared function on a Riemannian manifold.) For some regularity issues later, we further assume that the cost function is uniformly semiconcave on compact sets, i.e. for any compact coordinate charts $K \times \bar{K} \subset M \times \bar{M}$, there exists a smooth function $f: K \times \bar{K} \rightarrow \mathbf{R}$ such that $f+c$ is concave on $K \times \bar{K}$. Let $D \bar{D} c$ be the $n \times n$ matrix given by

$$
(D \bar{D} c)_{i \bar{j}}(x, \bar{x})=\frac{\partial^{2}}{\partial x^{i} \partial \bar{x}^{j}} c(x, \bar{x}) .
$$

On $N$ we will require that

$$
\operatorname{det}(D \bar{D} c) \neq 0
$$

and that $\bar{c}(\bar{x}, x):=c(x, \bar{x})$ both satisfy the twist (A1) condition: For each $x$,

$$
\bar{x} \rightarrow D c(x, \bar{x})
$$

is invertible, with an inverse depending continuously on $x$, and vice versa.

Now let $h_{c}$ be the pseudo-metric on $N \subset M \times \bar{M}$ that is defined at each $T_{(x, \bar{x})} N=$ $T_{x} M \times T_{\bar{x}} \bar{M}$ as

$$
h_{c}\left(\partial x^{i}, \partial \bar{x}^{j}\right)=h_{c}\left(\partial \bar{x}^{j}, \partial x^{i}\right)=\frac{\partial^{2}}{\partial x^{i} \partial \bar{x}^{j}} c(x, \bar{x})
$$


which is represented in coordinates by the nondegenerate symmetric matrix

$$
h_{c}=\left(\begin{array}{cc}
0 & -D \bar{D} c \\
-(D \bar{D} c)^{T} & 0
\end{array}\right)
$$

(This metric was introduced by the first and second author in [7].) Note that as a matrix, $h_{c}$ has $n$ positive and $n$ negative eigenvalues; in fact, we can choose coordinates so that $-D \bar{D} c$ is positive definite.

Given assumption (A1) together with some decay conditions on $\rho, \bar{\rho}$ if $M, \bar{M}$ are noncompact, we will always have a unique solution (see, for example, [13, Theorem $10.28])$ to the optimal transport problem, namely, a map $F: M \rightarrow \bar{M}$ which satisfies $F_{\#} \rho=\bar{\rho}$ in the sense of measures. One also has the unique solution $\gamma$ to the Kantorovich problem (c.f [13, Theorem 5.10]), that is a minimizing measure on $M \times \bar{M}$ which has marginals $\rho$ and $\bar{\rho}$. This measure $\gamma$ is associated to certain potential functions $u \in C(M)$ and $v \in C(\bar{M})$ in such a way that $\gamma$ is supported on $\Gamma$ defined as

$$
\Gamma=\{(x, \bar{x}) \in M \times \bar{M} \mid u(x)+v(y)-c(x, y)=0\} .
$$

The optimal mapping $F_{u}: M \rightarrow \bar{M}$ and inverse mapping $F_{v}: \bar{M} \rightarrow M$ which solve the symmetric optimal transport problems are derived from these potentials $u$ and $v$. The set $\Gamma$ contains the graph of both maps $F_{u}$ and $F_{v}$ (or what is sometimes called the graph of $F_{u}$ and the antigraph of $F_{v}$ ). One may regard $\Gamma$ as a multi-valued map either from $M$ or $\bar{M}$. It is a well-known fact that $\Gamma$ is single-valued exactly where $u$ (or $v$ ) is differentiable (in fact $C^{1}$-differentiable), and vice versa. Related to this, the following is also well-known:

Proposition 3.1. (c.f. [3] [10]) Both $F_{u}$ and $F_{v}$ differentiable almost everywhere.

Here is a sketch of the proof: The cost function $c$ is semi-concave, which gives semiconvexity of the $c$-convex solution $u$. (Using the conventions in [13, Theorem 5.2].) Then by Alexandrov's theorem, $u$ is twice differentiable almost everywhere, and together with assumptions (A1) (A2) we see that the transport maps must be differentiable where $u$ is so.

3.1. Choosing orientation and coordinates. We are assuming that $M$ comes with an orientation, which we respect. At any point $(x, \bar{x}) \in N, D \bar{D} c$ is nondegenerate, so by a change of coordinates (only on $\bar{M}$ ) we can arrange that $-D \bar{D} c$ is positive definite. This locally fixes an orientation on $\bar{M}$, which applies locally in $N$. In particular, it is possible that the orientations on the same neighborhoods on $\bar{M}$ may differ, depending on where they lie with respect to the product manifold. Fortunately, it is easy to check that on connected components of $N$, the (A2) condition implies that our choice of coordinates on $\bar{M}$, and hence orientation, is consistent.

Claim 3.2. Choosing coordinates on $\bar{M}$ so that $-D \bar{D} c+(-D \bar{D} c)^{T}$ is positive definite, the locally defined forms

$$
\tau=\frac{d x+d \bar{x}}{2}
$$

are consistent space orientation forms on $N$. 
Proof. Let $(x, \bar{x}) \subset \Omega \times \bar{\Omega}$ be any product coordinate chart, with $x$ consistent with the orientation on $M$. Let $\bar{x}=\varphi(\bar{y})$ for some diffeomorphism $\varphi: \bar{B} \rightarrow \bar{\Omega} \subset \bar{M}$. Then

$$
\partial_{y_{j}} \partial_{x_{i}} c(x, \varphi(\bar{y}))=\partial_{\bar{x}_{k}} \partial_{x_{i}} c(x, \bar{x}) \partial_{y_{j}} \varphi^{\bar{k}} .
$$

At a point, we are free to choose $\partial_{y_{j}} \varphi^{\bar{k}}$ to be anything (nondegenerate) that we want, so we choose it to be the inverse of $-\partial_{\bar{x}_{k}} \partial_{x_{i}} c(x, \bar{x})$. By smoothness of $c$ on $N$, $-\partial_{y_{j}} \partial_{x_{i}} c(x, \varphi(\bar{y}))$ will remain (after symmetrizing) positive definite on a nieghborhood on $N$, and hence on some subset of $\bar{A} \subset \bar{B}$. We declare the coordinates on $\bar{A}$ to be oriented, and $\Omega \times \bar{A}$ defines an orientation on $N$. One can repeat this construction at every point on $N$ and take a locally finite cover of such neighborhoods. These are consistent on any overlap, as both charts are defined to have differential close to the inverse of a smoothly changing matrix.

At this point, we fix a space orientation form $\tau$ by summing over a partition of unity. We also note that the coordinate definition 3.1 of $\Phi$ is now justified. For future reference, we note that

$$
\operatorname{det}(-\bar{D} D c)>0
$$

3.2. What happens where the transport map is smooth. Where differentiable,

the potential $u$ satisfies

$$
D u(x)=-D c\left(x, F_{u}(x)\right)
$$

(we are using conventions as in [13, Theorem 5.2]) and

$$
D^{2} u(x)=-D^{2} c\left(x, F_{u}(x)\right)-D \bar{D} c\left(x, F_{u}(x)\right) D F_{u}(x)
$$

in particular

$$
-D \bar{D} c\left(x, F_{u}(x)\right) D F_{u}(x)=D^{2} u(x)+D^{2} c\left(x, F_{u}(x)\right)
$$

the right-hand side of which is clearly symmetric. Further, $c$-convexity implies that

$$
D^{2} u(x) \geq-D^{2} c\left(x, F_{u}(x)\right) .
$$

To satisfy $F_{\#} \rho=\bar{\rho}$, the map $F_{u}$ must also satisfy, where differentiable

$$
\bar{\rho}\left(F_{u}(x)\right) \operatorname{det} D F_{u}(x)=\rho(x)
$$

recalling, (3.4). Now consider the following symplectic form on $M \times \bar{M}$ :

$$
\omega_{c}=\left(\begin{array}{cc}
0 & -D \bar{D} c \\
(D \bar{D} c)^{T} & 0
\end{array}\right),
$$

and the following conformal perturbation of $h_{c}$ :

$$
h^{\rho, \bar{\rho}}=\frac{1}{2}\left(\frac{\rho(x) \bar{\rho}(\bar{x})}{|\operatorname{det}(-D \bar{D} c)|}\right)^{\frac{1}{n}} h_{c} .
$$


First we show that the graph of $F_{u}$ is Lagrangian, wherever it is differentiable, by the following. Pull-back the form $\omega_{c}$ to $M$, and evaluate on any two tangent vectors

$$
\begin{aligned}
(I d \times F)^{*} \omega_{c}\left(\partial_{i}, \partial_{j}\right) & =\left\langle-D \bar{D} c\left(\partial_{i} F_{u}\right), \partial_{j}\right\rangle+\left\langle(D \bar{D} c)^{T} \partial_{i},\left(\partial_{j} F_{u}\right)\right\rangle \\
& =\left\langle-D \bar{D} c\left(\partial_{i} F_{u}\right), \partial_{j}\right\rangle+\left\langle\partial_{i}, D \bar{D} c\left(\partial_{j} F_{u}\right)\right\rangle \\
& =(D \bar{D} c D F)_{i j}-(D \bar{D} c D F)_{j i}
\end{aligned}
$$

which vanishes by (3.5).

Similarly, pulling back the metric $h_{c}$ :

$$
\begin{aligned}
(I d \times F)^{*} h_{c}\left(\partial_{i}, \partial_{j}\right) & =\left\langle-D \bar{D} c\left(\partial_{i} F_{u}\right), \partial_{j}\right\rangle+\left\langle(-D \bar{D} c)^{T} \partial_{i},\left(\partial_{j} F_{u}\right)\right\rangle \\
& =-(D \bar{D} c D F)_{i j}-(D \bar{D} c D F)_{j i}
\end{aligned}
$$

which is nonnegative by (3.6). The measure preserving condition (3.7), along with (3.6) and (3.5) guarantee that this metric will be strictly positive wherever the map is differentiable.

Proposition 3.3. Let $F: M \rightarrow \bar{M}$. Assume that $F$ is c-monotone, i.e.

$$
c(x, F(x))+c(y, F(y))-c(x, F(y))-c(y, F(x)) \leq 0 \quad \text { for all } x, y \in M .
$$

At any point of differentiability of $F$, the following holds

$$
(I d \times F)^{*} \Phi\left(\partial_{1}, \ldots, \partial_{n}\right)(x) \geq \sqrt{\operatorname{det} g_{i j}(x)}
$$

where

$$
g_{i j}=(I d \times F)^{*} h_{c}^{\rho, \bar{\rho}}\left(\partial_{i}, \partial_{j}\right)
$$

is the induced metric, with equality if and only if both (3.7) and (3.5) hold, in which case

$$
(I d \times F)^{*} \Phi\left(\partial_{1}, \ldots, \partial_{n}\right)(x)=\sqrt{\operatorname{det} g_{i j}(x)}=\rho(x) .
$$

Proof. First we compute the calibration, from (3.1)

$$
(I d \times F)^{*} \Phi\left(\partial_{1}, \ldots, \partial_{n}\right)=\frac{\rho+\operatorname{det} D F \bar{\rho}(F(x))}{2} .
$$

Next, using (3.8)

$$
\begin{aligned}
g_{i j} & =\frac{1}{2}\left(\frac{\rho(x) \bar{\rho}(F(x))}{|\operatorname{det}(D \bar{D} c)|}\right)^{\frac{1}{n}}\left(\begin{array}{cc}
I & (D F)^{T}
\end{array}\right)\left(\begin{array}{cc}
0 & -D \bar{D} c \\
-(D \bar{D} c)^{T} & 0
\end{array}\right)\left(\begin{array}{c}
I \\
D F
\end{array}\right) \\
& =\left(\frac{\rho(x) \bar{\rho}(F(x))}{|\operatorname{det}(D \bar{D} c)|}\right)^{\frac{1}{n}}\left(\frac{(D F)^{T}(-D \bar{D} c)^{T}+(-D \bar{D} c)(D F)}{2}\right) .
\end{aligned}
$$

Now taking the determinant

$$
\operatorname{det} g_{i j} \leq\left(\frac{\rho(x) \bar{\rho}(F(x))}{|\operatorname{det}(D \bar{D} c)|}\right) \operatorname{det}\left((D F)^{T}(-D \bar{D} c)^{T}\right)
$$


with equality if and only if $(D F)^{T}(-D \bar{D} c)^{T}$ is symmetric, recalling the fact (see [15], Lemma 3.1, also for a different formulation [11, pg 803] ) that for each $n \times n$ matrix $B$ with property $\langle B v, v\rangle \geq 0$ for every $v$,

$$
\operatorname{det}\left(\frac{1}{2}\left(B+B^{T}\right)\right) \leq \operatorname{det} B
$$

and the equality holds if and only if $B=B^{T}$. We show in Lemma 3.7 that due to $c$-monotonicity of $F$, the matrix $B=(D F)^{T}(-D \bar{D} c)^{T}$ satisfies $\langle B v, v\rangle \geq 0$ for every $v$.

In particular, applying Cauchy-Schwarz

$$
\sqrt{\operatorname{det} g_{i j}(x)} \leq \sqrt{\rho(x) \bar{\rho}(F(x)) \operatorname{det}(D F)} \leq \frac{\rho(x)+\bar{\rho}(F(x)) \operatorname{det}(D F)}{2}
$$

with equality if and only if (3.5) and (3.7) hold, respectively.

Corollary 3.4. The form $\Phi$ is a calibration.

Proof. The above calculation shows that this form has comass 1, noting that every spacelike plane can be obtained as the tangent space to the graph of a monotone function. Closedness of the form is clear.

3.3. Solutions defining a current. For a given optimal transport $F$, we consider the set $\Gamma$ given in (3.3), which is a closed set in $M \times \bar{M}$. It is well known that the set $\Gamma$ satisfies $c$-cylical monotonicity: if $\left(x_{i}, \bar{x}_{i}\right) \in \Gamma, i=1, \cdots, l$ then

$$
\sum_{i} c\left(x_{i}, \bar{x}_{i}\right) \leq \sum_{i} c\left(x_{i}, \bar{x}_{\sigma(i)}\right) \text { for any permutation } \sigma \text { of }\{1, \cdots, l\} .
$$

In particular, $F$ satisfies $c$-monotonicity. In our case we must deal with $\Gamma$ avoiding the cut-locus: Our arguments require that the current we are defining is compactly supported inside the region where the metric is smooth. If the metric is not smooth it may be difficult to define the comass of an $n$-form. Our main goal of this subsection is the following, which is similar in nature to the result in [10].

Proposition 3.5. Suppose that $c$ is smooth, and satisfies (A1) (A2) on a neighborhood of $\Gamma$. Then $\Gamma$ defines a current in a natural way.

Proof: We begin with the following, due to [10].

Proposition 3.6. Let $F$ be an optimal map and let $\Gamma$ be the set defined in (3.3). Suppose that $c$ is smooth and satisfies (A2) in a neighborhood of $\Gamma$. Then $\Gamma$ is $n$ rectifiable and locally finite.

The proof of this proposition is contained in [10]. We give the idea here, as we need the construction to define the current. Near any point $(x, \bar{x})$ in $\Gamma$ one can choose coordinates so that $D \bar{D} c=-I_{n}$ and $(x, \bar{x})$ is the origin. Near $(x, \bar{x})$ expand $c$ as a second order Taylor polynomial plus third order remainder. From the Taylor expansion together with $c$-monotonicity (3.9)

$$
c(x, \bar{x})+c(0,0) \leq c(x, 0)+c(0, \bar{x})
$$


one can show that

$$
x \cdot \bar{x} \geq O(3)
$$

so that for a small enough neighborhood $U$,

$$
|x+\bar{x}|=\sqrt{|x|^{2}+|\bar{x}|^{2}+2 x \cdot \bar{x}} \geq \sqrt{|x|^{2}+|\bar{x}|^{2}-O(3)}
$$

in particular the map $G: \Gamma \rightarrow \mathbb{R}^{n}$

$$
G(x, \bar{x})=x+\bar{x}
$$

is injective with Lipschitz inverse. Choose a compact $K \subset U$, then

$$
E:=G(\Gamma \cap K) \subset \mathbb{R}^{n}
$$

is a closed subset. One can compute that $G^{-1}$ is Lipschitz on $E$ and so extends to a Lipschitz map on an open set containing $E$. Now define the currents, first on $\mathbb{R}^{n}$

$$
S=\chi_{E} \vec{T}_{x} \mathbb{R}^{n}
$$

and then on $M \times \bar{M}$

$$
T_{K}=\left(G^{-1}\right)_{\#} S
$$

The result is a current on $M \times \bar{M}$ which is supported on the set $\Gamma \cap K$ and is represented by the tangent space to $\Gamma$ wherever it exists, which is almost everywhere $d \mathcal{H}^{n}$.

Observe that

$$
G^{*} d x=d x+d \bar{x}
$$

which, for our purposes, is the space orientation form. It follows that the tangent spaces to $\left(G^{-1}\right)_{\#} S$ are appropriately oriented, given the discussion in subsection 3.1. In particular there will be no cancellation when summing over a partition of unity. Given any precompact neighborhood $U$ in $M \times \bar{M}$ we can cover $\Gamma \cap U$ with finitely many open sets chosen as above. Taking a (locally finite) partition of unity, we build the current $T$.

Now that we have an integral current, we can write $T$ as

$$
T(\eta)=\int_{\Gamma}\langle\eta(x), \xi(x)\rangle d \mathcal{H}^{n}
$$

where $\xi(x)$ is an $\mathcal{H}^{n}$ almost everywhere simple unit tangent $n$-vector to $\Gamma$. 
3.4. The current $T$ is calibrated. Before we continue we need the following,

Lemma 3.7. Let $\Gamma \subset M \times \bar{M}$ be any c-monotone set. If $V+\bar{V}$ is a vector (decomposed in the obvious way) in the tangent space to $\Gamma$ at $\left(x_{0}, \bar{x}_{1}\right)$ then

$$
-\left.\partial_{V} \partial_{\bar{V}} c\right|_{(x, \bar{x})=\left(x_{0}, \bar{x}_{1}\right)} \geq 0 .
$$

In particular, $V+\bar{V}$ is weakly spacelike.

Proof. Let $S(t)=(s(t), \bar{s}(t))$ be a path in $\Gamma$ with $s(0)=\left(x_{0}, \bar{x}_{1}\right)$ and $s^{\prime}(0)=V+\bar{V}$. By the monotonicity condition at $t=0$

First, at $t=0$

$$
\begin{aligned}
\frac{d}{d t}\left\{c(s(t), \bar{s}(t))+c\left(x_{0}, \bar{x}_{1}\right)-c\left(s(t), \bar{x}_{1}\right)-c\left(x_{0}, \bar{s}(t)\right)\right\} & = \\
D c \cdot s^{\prime}(0)+\bar{D} c \cdot \bar{s}^{\prime}(0)-D c \cdot s^{\prime}(0)-D \bar{c} \cdot \bar{s}^{\prime}(0) & =0
\end{aligned}
$$

and using the monotonicity condition at $t=0$

$$
\begin{aligned}
& \frac{d}{d t}\left\{c(s(t), \bar{s}(t))+c\left(x_{0}, \bar{x}_{1}\right)-c\left(s(t), \bar{x}_{1}\right)-c\left(x_{0}, \bar{s}(t)\right)\right\}= \\
& \quad D^{2} c\left(s^{\prime}(0), s^{\prime}(0)\right)+D c \cdot s^{\prime \prime}(0)+2 D \bar{D} c\left(s^{\prime}(0), \bar{s}^{\prime}(0)\right)+\bar{D}^{2} c\left(\bar{s}^{\prime}(0), \bar{s}^{\prime}(0)\right) \\
& +\bar{D} c \cdot \bar{s}^{\prime \prime}(0)-D^{2} c\left(s^{\prime}(0), s^{\prime}(0)\right)-D c \cdot s^{\prime \prime}(0)-\bar{D}^{2} c\left(\bar{s}^{\prime}(0), \bar{s}^{\prime}(0)\right)-\bar{D} c \cdot \bar{s}^{\prime \prime}(0) \leq 0
\end{aligned}
$$

in particular $2 D \bar{D} c(V, \bar{V}) \leq 0$.

Lemma 3.8. Suppose that $\xi$ is an n-vector representing the tangent space to the current $T$ at $x$, and $\eta$ is a form such that $\left\langle\eta_{x}, \xi\right\rangle<0$. Then $\eta$ has negative comass.

Proof. First, we note by construction, that the tangent planes are $\tau$-oriented. If $\xi$ is strictly spacelike, the result follows from definition 2.1 by scaling $\xi$ to unit size. So suppose that $\xi$ is only weakly spacelike (this is the case be Lemma 3.7). Rotating coordinates, we consider the metric in the form $d x^{2}-d y^{2}$. Take a basis for $\xi$, say $\left\{e_{i}\right\}$, and note that for each $e_{i}$,

$$
d x^{2}\left(e_{i}, e_{i}\right) \geq d y^{2}\left(e_{i}, e_{i}\right) \geq 0
$$

and $d x^{2}\left(e_{i}, e_{i}\right)$ is strictly positive for some $i$. Now we make a perturbation of each $e_{i}$ by scaling it slightly in the $x$ component. The plane spanned by these vectors $\xi_{\varepsilon}$, is then strictly spacelike. By continuity, we conclude that $\langle\eta, \xi\rangle<0$ for some strictly spacelike plane, so has negative comass.

Claim 3.9. The infimum in the definition of mass for the current $T$ is attained by the calibration $\Phi$.

Proof. Assume not. There exists a form $\varphi$ of comass 1 with the property that $T(\varphi)<T(\Phi)$. By the Riesz Representation

$$
\int_{\Gamma}\langle\varphi(x)-\Phi(x), \xi(x)\rangle d \mathcal{H}^{n}<0
$$


so we conclude that there is a rectifiable set $\Upsilon \subset \Gamma$ with positive $\mathcal{H}^{n}$ measure such that on $\Upsilon$

$$
\langle\varphi(x), \xi(x)\rangle<\langle\Phi(x), \xi(x)\rangle .
$$

If $\Upsilon$ projects to a set of positive measure in either direction (i.e. either to $M$ or $\bar{M}$ ), we can conclude that it contains a point where the optimal transport map is differentiable, and we can draw a contradiction from Proposition 3.3: At points of differentiability the tangent planes are calibrated, it follows that

$$
\langle\Phi(x), \xi(x)\rangle=\|\xi\|_{h^{\rho, \bar{\rho}}} \leq\langle\varphi(x), \xi(x)\rangle
$$

because $\varphi$ has comass 1 . So we conclude that $\Upsilon$ is in the inverse image of sets of zero measure under both projections. It follows then the calibrating form (which measures the volumes of projections of a given plan in either direction) must vanish on $\xi$ : so in fact we have $\langle\varphi(x), \xi(x)\rangle<0$. But this contradicts Lemma 3.8.

This leads to our main theorem.

Theorem 3.10. Suppose that $\rho, \bar{\rho}$ are smooth densities, and that $c$ is smooth and satisfies (A1) (A2). Suppose the minimizing measure $\gamma$ is compactly supported away from the cut locus. Then the spacelike current $T$ defined on $\operatorname{spt}(\gamma)$ is homologically mass maximizing: $\|T\|_{\left(N, h^{\rho, \bar{\rho}}\right)} \geq\|S\|_{\left(N, h^{\rho, \bar{\rho}}\right)}$ for all compactly supported $n$-currents $S$ which are homologous to $T$.

Proof. By the previous claim, we have $\|T\|_{h^{\rho, \bar{\rho}}}=\Phi(T)$. If $S$ is homologous to $T$ then by definition of mass (2.1) $\|S\|_{h^{\rho, \bar{\rho}}} \leq \Phi(S)=\Phi(T)$, because $\Phi$ has comass 1 by Corollary, 3.4.

We note further that as calibration arguments require that we work with currents of compact support, for many situations the result does not directly apply globally (if $M$ is not compact), however it will apply locally. Note that given any optimal map $F: M \rightarrow \bar{M}$ the restriction of the optimal map to any subset is an optimal map onto its image, and satisfies the same relations (3.6) (3.5) and (3.7), hence locally is calibrated.

We can now compare a smooth space-like submanifold to smooth variations of the submanifold, and we conclude:

Corollary 3.11. At any point where the graph of the optimal transport map $F$ is a $C^{1}$ strictly space-like submanifold, the graph has zero mean curvature as a submanifold of $\left(M \times \bar{M}, h_{c}^{(\rho, \bar{\rho})}\right)$. 


\section{Further Remarks}

Remark 4.1. We see that the graph of a map $F$ which gives a calibrated submanifold satisfies both (3.5) and (3.7). In particular, (3.5) means that $F$ is given locally by the cost exponential of a potential function $u$. If the function $u$ is global, then the map $F$ is optimal. If the function $u$ is only local, then $F$ is not a cost minimizer, although it solves the volume maximization problem. The existence of such solutions is shown in [14].

Remark 4.2. We also mention a remark on the Ma-Trudinger-Wang cost-curvature condition [9, A3 condition]. The first two authors [7] expressed the MTW condition as a curvature condition on $h_{c}$, which may be described as follows: The weak (strong) MTW A3 condition holds if and only if, in each coordinate chart on $M$ and $\bar{M}$, the Riemannian sectional curvature $R_{i \bar{j} \bar{j} i}$ corresponding to any vanishing component $\left(h_{c}\right)_{i \bar{j}}=0$ of the metric tensor is nonnegative (positive).

With respect to the conformal metric $h^{\rho, \bar{\rho}}$, we have the Riemann curvature tensor

$$
R_{i \bar{j} \bar{j} i}^{\rho, \bar{\rho}}=\left(\frac{\pi^{*} \rho \wedge \bar{\pi}^{*} \bar{\rho}}{d \operatorname{vol}_{h_{c}}}\right)^{\frac{1}{n}}\left(R_{i \bar{j} \bar{j} i}+\Lambda_{i \bar{j}}\left(h_{c}\right)_{\bar{j} i}-\Lambda_{i i}\left(h_{c}\right)_{\bar{j} \bar{j}}+\Lambda_{\bar{j} i}\left(h_{c}\right)_{i \bar{j}}-\Lambda_{\bar{j} \bar{j}}\left(h_{c}\right)_{i i}\right)
$$

for some $\Lambda_{i j}$ involving derivatives of the conformal factor. For metric components $\left(h_{c}\right)_{i \bar{j}}=0$, we easily see the corresponding component of the Riemann tensor is given by

$$
R_{i \bar{j} \bar{j} i}^{\rho, \bar{\rho}}=\left(\frac{\rho(x) \bar{\rho}(F(x))}{|\operatorname{det}(D \bar{D} c)|}\right)^{1 / n} R_{i \bar{j} \bar{j} i} .
$$

Thus, the weak (strong) MTW A3 condition holds if and only if, in each coordinate chart on $M$ and $\bar{M}$, whenever $h_{i \bar{j}}{ }^{\rho, \bar{\rho}}=0$, the sectional curvature $R_{i \bar{j} \bar{j} i}^{\rho, \bar{p}}$ is nonnegative (positive).

Remark 4.3. Much the above may be phrased quite elegantly in the language of split special Lagrangian geometry of Harvey and Lawson. In [4] the calibration (1.4) is seen to be the real part of a $\mathbb{D}$-holomorphic volume form, and the conformal factor on the metric (1.3) gives this holomorphic form constant length.

\section{Acknowledgements}

The authors are thankful to Reese Harvey and the referee, who pointed out the reference [11] as well as other helpful suggestions, also to Blaine Lawson for his interest, helpful discussions, and for sending the preprint [4], and to Christina Sormani for helpful suggestions.

\section{References}

[1] G. Alberti and L. Ambrosio, A geometrical approach to monotone functions in $\mathbf{R}^{n}$, Math. Z. 230 (1999), no. 2, 259-316.

[2] P. Delanoë, Lie solutions of Riemannian transport equations on compact manifolds, Differential Geom. Appl. 26 (2008), no. 3, 327-338. 
[3] W. Gangbo and R. J. McCann, The geometry of optimal transportation, Acta Math. 177 (1996), no. $2,113-161$.

[4] R. Harvey and H. B. Lawson, Jr., Split special Lagrangian geometry, arXiv:1007.0450v1.

[5] - Calibrated geometries, Acta Math. 148 (1982) 47-157.

[6] N. J. Hitchin, The moduli space of special Lagrangian submanifolds, Ann. Scuola Norm. Sup. Pisa Cl. Sci. (4) 25 (1997), no. 3-4, 503-515 (1998). Dedicated to Ennio De Giorgi.

[7] Y.-H. Kim and R. J. McCann, Continuity, curvature, and the general covariance of optimal transportation, J. Eur. Math. Soc. (JEMS) 12 (2010) 1009-1040.

[8] G. Loeper, On the regularity of solutions of optimal transportation problems, Acta Math. 202 (2009), no. 2, 241-283.

[9] X.-N. Ma, N. S. Trudinger, and X.-J. Wang, Regularity of potential functions of the optimal transportation problem, Arch. Ration. Mech. Anal. 177 (2005), no. 2, 151-183.

[10] R. J. McCann, B. Pass, and M. Warren, Rectifiability of optimal transportation plans, arXiv:1003.4556v1.

[11] J. Mealy, Volume maximization in semi-Riemannian manifolds, Indiana Univ. Math. J. 40 (1991), no. 3, 793-814.

[12] N. S. Trudinger and X.-J. Wang, On the second boundary value problem for Monge-Ampère type equations and optimal transportation, Ann. Sc. Norm. Super. Pisa Cl. Sci. (5) 8 (2009), no. $1,143-174$

[13] C. Villani, Optimal transport, Vol. 338 of Grundlehren der Mathematischen Wissenschaften [Fundamental Principles of Mathematical Sciences], Springer-Verlag, Berlin (2009), ISBN 9783-540-71049-3. Old and new.

[14] M. Warren, A McLean theorem for the moduli space of Lie solutions to mass transport equations, Arxiv: 1006.1334 .

[15] — Calibrations associated to Monge-Ampère equations, Trans. Amer. Math. Soc. 362 (2010), no. 8, 3947-3962.

Department of Mathematics, University of British Columbia, Vancouver BC Canada and Institute for Advanced Study, Princeton NJ USA.

E-mail address: yhkim@math.ubc.ca

Department of Mathematics, University of Toronto, Toronto, Ontario Canada.

E-mail address: mccann@math.toronto.edu

Department of Mathematics, Princeton University, Princeton NJ, USA

E-mail address: mww@princeton.edu 